Sädhanā, Vol. 18, Part 5, September 1993, pp. 843-856. (C) Printed in India.

\title{
Low frequency oscillations in power systems: A physical account and adaptive stabilizers
}

\author{
D P SEN GUPTA and INDRANEEL SEN \\ Department of Electrical Engineering, Indian Institute of Science, \\ Bangalore 560 012, India
}

\begin{abstract}
This paper presents a physical explanation of the phenomenon of low frequency oscillations experienced in power systems. A brief account of the present practice of providing fixed gain power system stabilizers (PSS) is followed by a summary of some of the recent design proposals for adaptive PSS. A novel PSS based on the effort of cancelling the negative damping torque produced by the automatic voltage regulator (AVR) is presented along with some recent studies on a multimachine system using a frequency identification technique.
\end{abstract}

Keywords. Power system stability; frequency identification; adaptive stabilizers.

\section{Introduction}

Hunting in synchronous generators experienced in the early thirties was caused by the negative damping generated in the armature and in transmission lines (Kron 1952). Field winding was the only source of positive damping at that time. Provision of amortisseur windings helped to overcome the problem of small amplitude, low frequency oscillations, which reappeared in the sixties with the introduction of high gain, low time constant automatic voltage regulators and transmission of bulk power over long transmission lines. Intertie oscillations were reported in SaskatchewanManitoba-Ontario West interconnections by Hanson et al (1968). Similar oscillations are being experienced by various other utilities such as the Central Electricity Generating Board, UK (see Gibson 1988). In India, low frequency power oscillations were repeatedly experienced in the Talcher-Balimela line linking the southern and eastern regions of India. A $210 \mathrm{MW}$ thermal unit in Raichur, Karnataka, which was linked through a long tie line $\left(X_{e} \approx 0.9\right.$ p.u.) could not deliver the rated power, since low frequency power oscillations would set in as soon as the generator was loaded beyond $160 \mathrm{MW}$.

A remedy to this problem has been sought using a power system stabilizer (PSS) in the form of excitation control. A suitable signal such as change in angular velocity $(\Delta \omega)$, accelerating power $(\Delta P)$, change in frequency $(\Delta f)$ or a combination of these signals is suitably phase-shifted, amplified and fed into the AVR circuit.

A classic paper (deMello \& Concordia 1969) on the subject, using frequency response techniques provides a thorough analysis of the phenomenon of small oscillation and 
describes the effects of a PSS. Byerly et al (1982) have carried out extensive studies on the subject and used eigenvalue techniques for studying the performance of PSS in a multimachine complex. A practical method of tuning a PSS has been presented in a three-part paper by Larsen \& Swann (1981) and also by Farmer \& Agrawal (1983). Although power systems stabilizers, by and large, have alleviated the problem of low frequency oscillations, the tuning of a PSs still presents difficulties. A low frequency $(0.7 \mathrm{~Hz})$ oscillation is still experienced in the US, north of Arizona, and the utilities find it difficult to cope with this problem.

Since power system dynamics keeps changing continuously with changes in active and reactive power transport and changes in the topology of the transmission system, fixed gain stabilizers may not be able to cope with all situations. The problem is exacerbated when a number of generators with different inertia are called upon to operate on the brink of their dynamic stability. Major manufacturers like Brown Boveri and Siemens have introduced variable gain power system stabilizers. Several authors have used the theory of adaptive control and proposed adaptive power system stabilizers. The present authors have approached the problem differently. Having developed a simple method of isolating the negative damping torque contributed by the AVR, a simple algorithm has been proposed by Sen Gupta et al (1985) to cancel the negative damping, the magnitude of which changes with operating and system conditions.

This paper briefly reviews some of the adaptive or gain scheduling stabilizers proposed, elaborating on the decomposition of damping torque and the PSS based on the cancellation of negative damping. The multiple frequencies of oscillation that a generator may experience in a multimachine system are identified and the information is used in the design of the PSS. The next section presents the decomposition of the total damping torque generated into its various components, in an attempt to understand the physical process that makes a generator undergo small oscillations.

\section{Causes leading to small oscillations}

Four major factors or their combinations are known to lead to poor damping. These are: (a) large active power, (b) large negative reactive power, (c) large AVR gain with low time constant, and (d) weak ties or long tie lines $\left(X_{e}>0.7\right.$ p.u). For small oscillation stability the following modes are of relevance.

(a) Intraplant oscillations $(2-3 \mathrm{~Hz})$. These oscillations are confined within a plant with the generators oscillating against each other. This is not very common and the PSS is not designed to cope with this mode.

(b) Local mode $(0.8-1.6 \mathrm{~Hz})$ when a synchronous generator oscillates against a large system (a single machine infinite bus configuration).

(c) Interarea mode $(0.2-0.7 \mathrm{~Hz})$ when a group of generators in an area linked by a long tie line oscillates against a group in another area.

(d) Exciter mode $(1.5-2.5 \mathrm{~Hz})$ oscillations confined within the exciter loop, called electrical or megavar oscillations. This mode may be excited by ill-tuned stabilizers.

\subsection{Components of damping torque}

It is well-known that damping in a synchronous machine is caused by additional copper loss produced by the oscillatory currents in the windings. Quantification of 
these components, however, presented difficulties. Synchronous generators are normally analysed from Park's reference frame which are rigidly connected to the rotor axes (field axes). Changes in currents in the field and amortisseur windings obviously are true or absolute changes. The changes in the armature currents/voltages are apparent since the observer oscillates, with reference to the armature and any attempt to relate damping and copper loss proves unsuccessful (Sen Gupta et al 1977; Sen Gupta \& Lynn 1980). Covariant differentials are computed to provide true changes $(\delta i)$ and $\left(\delta i^{2} R\right)$ gives a correct measure of damping in a winding of resistance $R$. Although the relation between damping in each winding and the copper loss could be established only by rigorous analysis using principles of tensor invariance, the following relations quantify very accurately the damping torque contributions from the rotor windings.

Damping contribution from field winding,

$$
T_{D f}=\Delta i_{f}^{2} R_{f} h^{2}
$$

Damping contribution from $q$-axis amortisseur winding,

$$
T_{D k q}=\Delta i_{k q}^{2} R_{k q} / h^{2} \text {. }
$$

Damping contribution from $d$-axis amortisseur winding,

$$
T_{D k d}=\Delta i_{k d}{ }^{2} R_{k d} / h^{2}
$$

The sum of these constitutes damping from rotor windings, which is always positive. Armature damping is negligible. The main source of negative damping is the AVR circuit. The contributions of the AVR to damping $\left(T_{D r}\right)$ and synchronizing torque $\left(T_{S r}\right)$ have been quantified (Sen Gupta et al 1977) by the simple relationships below:

$$
\begin{aligned}
& T_{D r}=\operatorname{Real}\left(K_{A} \Delta V_{t} \Delta i_{f}^{*} / h^{2}\right)=K_{A} \Delta V_{t} \Delta i_{f} \cos \phi / h^{2} \\
& T_{S r}=\operatorname{Imag}\left(K_{A} \Delta V_{t} \Delta i_{f}^{*} / h\right)=K_{A} \Delta V_{t} \Delta i_{f} \sin \phi / h
\end{aligned}
$$

$K_{A}$ is the AVR gain. The AVR time constant is neglected. The incremental voltages and currents are obtained from Park's incremental equations.

$$
[\Delta V]=[Z][\Delta i]
$$

Sinusoidal oscillations are assumed and the p-operator in the $[Z]$ matrix is replaced by $j h \omega$ where $h \omega$ is the hunting frequency. Elements of $[\Delta i]$, the oscillating current vector, represent currents in each winding and are obtained from

$$
[\Delta i]=[Z]^{-1}[\Delta V] \text {. }
$$

Of all the components of damping torque [computed from the relations (1)-(4)], the contributions from the $q$-axis amortisseur winding and from the AVR circuit are the most critical as will be seen presently. Figures 1 and 2 represent different torque components when reactive power $Q$ and the line reactance $X_{e}$, respectively, are varied.

In figure 1 , the total damping torque $T_{D \ell}$ depends largely on $T_{D k q}$ and $T_{D r}$. As the reactive power becomes zero and then negative, $T_{D k q}$ reduces sharply and $T_{D r}$ becomes negative. The same thing happens when $X_{e}$ is increased (for a constant value of $P$ ). The reduction in positive damping from the $q$-axis amortisseur winding and the injection of negative damping from the AVR happen at the same time, making the generator prone to hunting. 


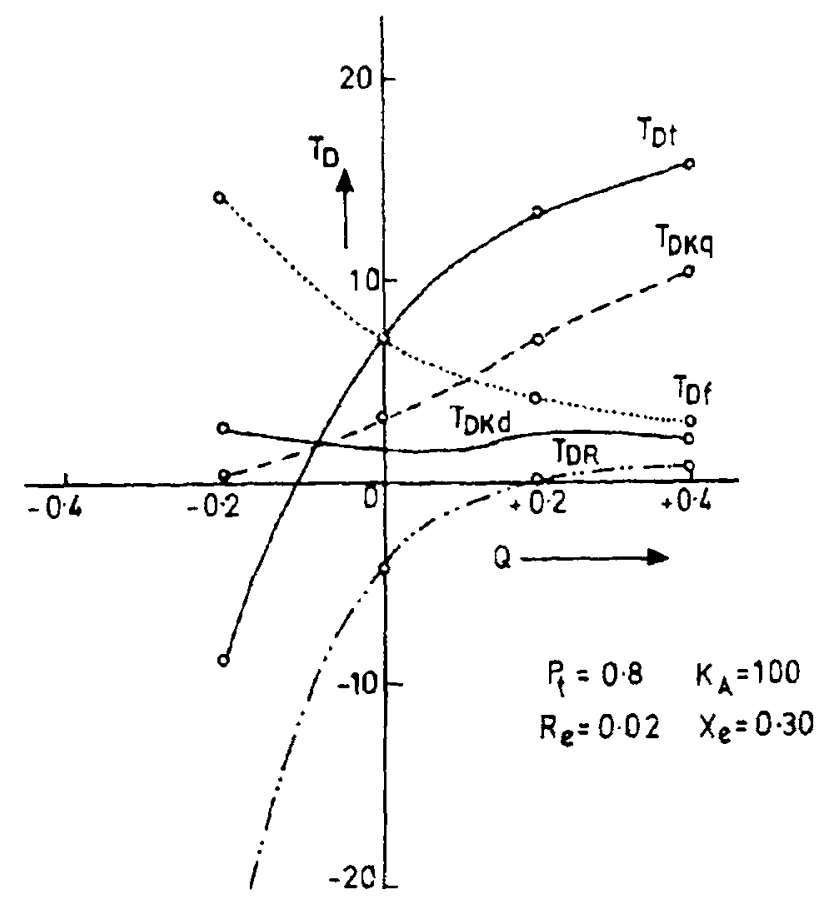

Figure 1. Comporents of damping torque varying with reactive power. $T_{D k_{q}}=$ damping contribution from $q-$ axis amortisseur winding; $T_{D r}=$ damping contribution Irom AVR.

Simple quantitative relations derived by Nambiar (1984) show that

and

$$
T_{D k q} \propto \cos ^{2} \delta
$$

$$
T_{D k q} \propto 1 /\left(X_{e}+Z^{*}\right)^{2},
$$

where $\delta$ is the load angle and $Z^{*}$ is the transfer impedance viewed inside from the generator terminal. These relations clearly show how $T_{D k q}$ gets depleted with an

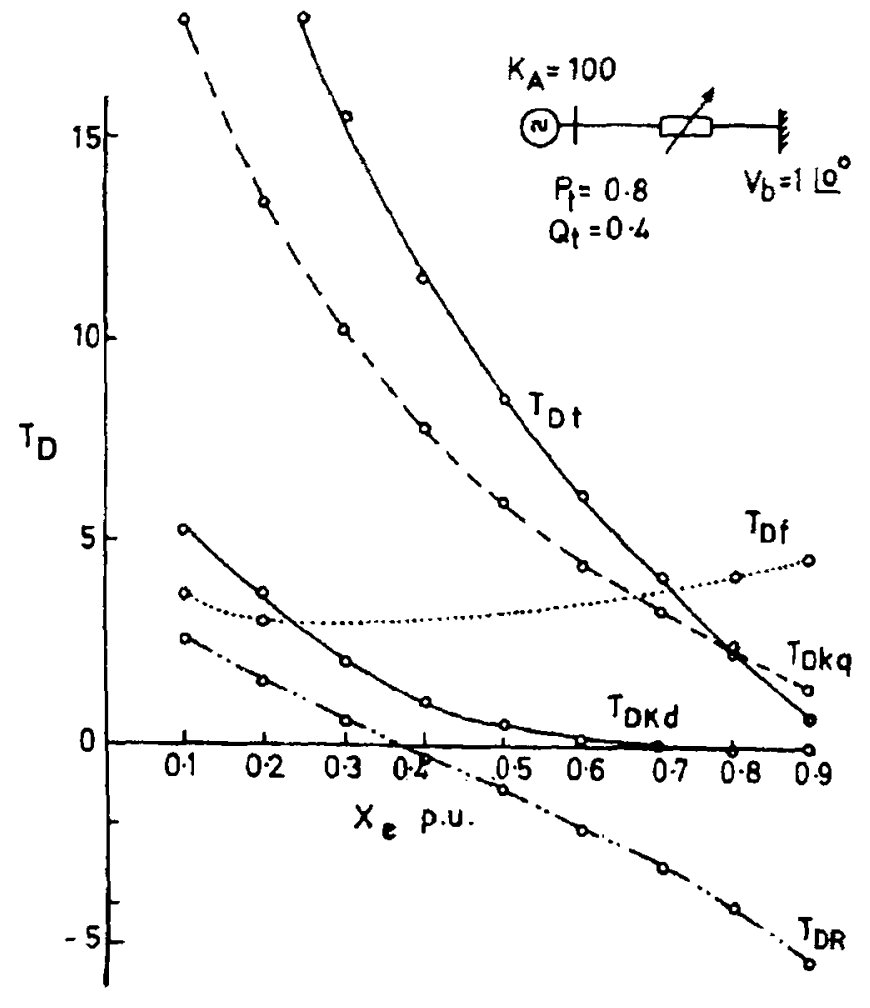

Figure 2. Components of damping torque varying with line reactance. 
increase of $P$ and $-Q$, both of which increase the load angle $\delta . T_{D k q}$ decreases steeply with the increase of $X_{\mathfrak{e}}$.

The angle $\phi$ in (4) represents the phase relation between $\Delta V_{\mathrm{t}}$ and $\Delta i_{f}$. Under most operating conditions $\phi>90^{\circ}$. It is evident that the AVR gain $K_{A}$ directly multiplies $T_{D r}$ and if $\phi>90^{\circ}$, the negative damping contribution from the AVR increases almost linearly with $K_{A}$. This explains why large AVR gain leads to dynamic instability. In the early years, AVR seldom gave rise to instability because the time constant was large. If the time constant is $T_{A}$ and the frequency of oscillation is $j h \omega$, the AVR contribution to damping is

$$
T_{\mathrm{Dr}}=K_{A} \Delta V_{t} \Delta i_{f} \cos (\phi-\theta),
$$

where $\theta=\tan ^{-1}\left(\omega h T_{A}\right)$. If $T_{A}$ is large $\phi-\theta$ is more likely to be an acute angle and $T_{D r}$ may be positive.

The power system stabilizer proposed by the authors cancels the negative damping torque generated in the AVR circuit. This has been reported earlier (Sen Gupta et al 1985). Experimental results obtained subsequently are briefly mentioned later in this paper with indications of how we propose to extend this to a multimachine system.

\section{Adaptive power system stabilizer}

Deita-omega $(\Delta \omega)$ or speed signal was used for a long time as a stabilizing signal. In view of the inherent lag $(\phi)$ of the AVR circuit, the signal needs to be phase advanced. A lead/lag circuit is provided for the purpose (figure 3 ) but the setting of time constants $T_{1}-T_{4}$, measurement of the $\Delta \omega$ signal and filtering out of subsynchronous oscillations present problems. An accelerating signal is generally favoured now for a PSS and it is synthesized from electrical measurements. It is common practice to use two signals such as $\Delta P$ and $\Delta P / s$ (which is approximately proportional to $\Delta \omega$ and orthogonal to a $\Delta P$ signal) and to multiply these signals by two gains $k_{1}$ and $k_{2}$. The advantage of using two signals is that by adjusting the amplitudes of $k_{1}$ and $k_{2}$, the amplitude and phase of the PSS signal can be easily controlled.

Although Kundur et al (1989) seem to achieve good performance using fixed gain stabilizers designed on the basis of extensive system analysis and consider variable

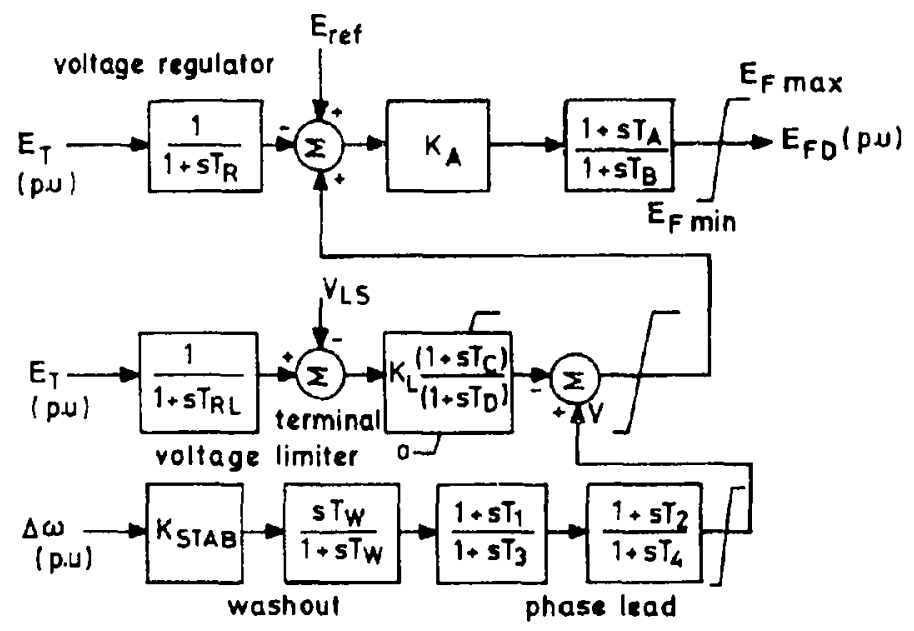

Power System Stabilizer

Figure 3. Excitation systems with $\Delta \omega$ power system stabilizer. 
gain PSS to be unnecessary, others do not share their views. One of the major factors that may come in the way of an exhaustive system analysis is the non-availability of data for all generators, transmission systems and in particular of the loads in a large power system. Even information on AVR gains and time-constants, crucial for dynamic stability studies, may not be readily available.

It seems to be more realistic to try to "observe" the rest of the system from a generator and represent it by a simple model (Grund 1978). The Model Reference Adaptive Controller (MRAC) and Self-tuning Regulator (STR) are briefly described below as also the PSS proposed by the authors which essentially follow the same principle of observing the system from the generator for which the PSS is being designed. Malik and Hope (see Kanniah et al 1984), Hogg (see .Wu \& Hogg 1990) and their co-workers have used model reference adaptive control (MRAC) and selftuning regulators in particular for PSS design for a single-machine infinite bus (SMIB) model and then extended the studies to multimachine systems. The essential features of MRAC and STR are summarized below.

\subsection{Model reference adaptive control}

In model reference adaptive control the aim is to make the output of an unknown plant asymptotically approach the output of a given reference model. The reference model represents the desired plant behaviour. The specified performance is compared with the actual performance of the unknown plant. The plant and the reference system are excited by the same input to generate an error signal which is then used to adjust the parameters of a suitable controller or to generate an auxiliary input signal to minimize the error.

The signal synthesis adaptation involves the adjustment of only input variables, whereas parameter adjustment requires real-time adjustment of a few plant parameters. In either case the MRAC control scheme involves three steps.

(1) Setting up an appropriate reference model retaining the essential dynamic behaviour of the actual system.

(2) Computation of error between the desired and actual output response.

(3) Using the correlation between this error and the system states, either existing controller parameters are adjusted or an auxiliary feedback control signal is synthesized in order to minimize the error.

\subsection{Self-tuning regulators}

The seif-tuning regulator is another form of an adaptive controller. In STR, a design procedure for the known plant parameters is first chosen. This is applied to the unknown plant using recursively estimated values of the parameters. As the parameters are recursively estimated the unknown plant is driven to produce the desired response.

The STR design is essentially a stochastic control problem. Based on the 'certaintyequivalence-principle' a set of system parameters are identified and the control is calculated using a prescheduled strategy. The essential design steps are:

(1) formulation of an appropriate equivalent model for the system to be controlled;

(2) identification of the model parameters at each sampling instant. This step generally requires persistent random noise injection into the system;

(3) on-line design of the controller with the help of the identified parameters. 
A number of different strategies can be employed for identification as well as the design of the controller.

\subsection{Gain scheduling controller}

The third method of PSS design is based on gain scheduling controllers (GSC). In these controllers, the gains of the regulator are varied according to some prespecified control strategy depending upon the plant operating conditions. The gain computations can be either off-line or on-line. The off-line computations of gains reduce real-time processing and, therefore, these stabilizers are well suited for practical applications. The stabilizers are also comparatively more robust than the other stabilizers discussed earlier.

The GSC are designed based on diverse mathematical formulation of the system.

To the best of the authors' knowledge, the PSS based on MRAC and STR have not been used in actual power systems. The variable gain PSS that have been utilized so far have been manufactured by Brown Boveri and Siemens.

The Brown Boveri variable gain PSS is essentially based on a look-up table. As has been stated earlier, damping torque depends mainly on $P, Q$, and $X_{e}$. For three values each, suboptimal gains $k_{1}, k_{2}$ to be associated with $\Delta P$ and $\Delta P / s$ (or $(\Delta f)$ signals are computed off-line for two values of $X_{e}$ and stored in a microprocessor. The computation is based on a single machine infinite bus model (SMIB) with tie line reactance of $X_{e}$ and $D$-partition technique is employed for obtaining $k_{1}$ and $k_{2}$. For a certain value of $P, Q$ and $X_{e}$, the corresponding values of $k_{1}$ and $k_{2}$ are recalled from memory and used in the PSS. Whereas $P$ and $Q$ are easily measured, $X_{e}$ cannot be measured very easily. It is identified by measuring the frequency of oscillations when it sets in. If the frequency is high, the system is deemed as strong ( $X_{\mathrm{e}} \approx 0.4$ p.u.). If the frequency is low the system is deemed to be weak. (Frequency of oscillation $\omega h=\left(T_{s} / M\right)^{1 / 2}$ where $T_{s}$ is the synchronizing torque which decreases as $X_{e}$ is increased. $M$ is the inertia constant.) The main limitation of this method is that the off-line computations are carried out on an SMIB configuration and can cope with the local mode only.

Siemens synthesize the $\triangle P$ signal and split it into two sets of orthogonal components to feed into a PSS after multiplying the components by suitable gains.

3.3a A PSS to cancel negative damping: It has been shown by Narahari (1982) that the terminal voltage oscillation $\Delta V_{t}$ can be expressed as

where

$$
\Delta V_{t}=k_{f} \Delta i_{f}+k_{\omega} \Delta \omega+k_{\delta} \Delta \delta
$$

$$
\begin{aligned}
& k_{f}=X_{e} X_{a d}\left(1-X_{a d} / X_{k d}\right) \cos \left(\delta_{t}\right) /\left(X_{d}^{*}+X_{e}\right) . \\
& k_{\omega}=\left[V_{q t} /\left(X_{d}^{*}+X_{e}\right) \times\left\{E_{f \dot{d}}-\left(X_{d}-X_{d}^{*}\right) i_{d}\right\}\right. \\
& \left.\quad+V_{d t}\left(X_{q}-X_{q}^{*}\right) i_{q} /\left(X_{q}^{*}+X_{e}\right)\right] X_{e} /\left(V_{t} \omega_{0}\right), \\
& k_{\delta}=\left[V_{d t} X_{d}^{*} \cos \left(\delta_{t}\right) /\left(X_{d}^{*}+X_{e}\right)\right. \\
& \left.\quad-V_{q t} X_{d}^{*} \sin \left(\delta_{t}\right) /\left(X_{q}^{*}+X_{e}\right)\right] V_{b} / V_{t},
\end{aligned}
$$

where $X_{d}^{*}=X_{d}-X_{a d}^{*} / X_{k d}$ and $X_{q}^{*}=X_{q}-X_{a q}^{*} / X_{k q}$, other terms having their usual meanings. 
Replacing $\Delta \delta$ by $\Delta P$ signal and simplifying these expressions for turboalternators $\left(X_{d}=X_{q}, X_{a d}=X_{a q}, X_{k d}=X_{k q}\right)$, adaptive gains used for studies on a microalternator and the thermal power plant are

$$
\begin{aligned}
& k_{P}=C P_{t} / M V_{t} h^{2} \omega^{2}, \\
& k_{\omega}=\left[V_{t} / X_{d}^{*}+Q_{t} / V_{t}\right] C / \omega .
\end{aligned}
$$

These gains can be easily computed by analogue circuits.

Multiplying both sides of (11) by $\Delta_{f}^{*}$, the complex conjugate of $\Delta i_{S}$

$$
\Delta V_{t} \Delta i_{f}^{*}=k_{f} \Delta i_{f} \Delta i_{f}^{*}+k_{\omega} \Delta \omega \Delta i_{f}^{*}+k_{\delta} \Delta \delta \Delta i_{f}^{*} .
$$

Since $k_{f}$ is positive for most operating conditions and $\Delta i_{f} \Delta i_{f}^{*}$ is positive, $k_{f} \Delta i_{f} \Delta i_{f}^{*}$ is almost always positive. The negative damping contribution from $\Delta V_{l} \Delta i_{f}^{*}$, is obviously from $k_{\omega} \Delta \omega \Delta i_{f}^{*}+k_{\delta} \Delta \delta \Delta i_{f}^{*}$. It follows from (12) that

$$
\Delta V_{\imath}-k_{\omega} \Delta \omega-k_{\delta} \Delta \delta=k_{f} \Delta i_{f},
$$

will contribute positive damping torque when the PSS signal is $-\left(k_{\omega} \Delta \omega+k_{\delta} \Delta \delta\right)$. Since $\Delta \delta$ is not easy to measure $k_{\delta} \Delta \delta$ may be replaced by $k_{p} \Delta P,\left(-M h^{2} \omega^{w} \Delta \delta \approx \Delta P\right)$.

Extensive computation has confirmed this theory. Experiments on a laboratory model power system using a microalternator have shown excellent agreement with theoretical studies. These studies were all based on a single machine infinite bus (SMIB) configuration. The synchronous machine exhibited only the local mode.

Experiments have been carried out on a $210 \mathrm{MW}$ thermal unit at Mettur, Tamil Nadu. A disturbance was initiated by a $5 \%$ step input into the AVR circuit and an oscillation of $1.4 \mathrm{~Hz}$ was recorded. The oscillation was obviously of the local mode with the $210 \mathrm{MW}$ generator oscillating against the entire Tamil Nadu system. The oscillation using our PSS gave distinctly better damping than the fixed gain PSS provided by the manufacturer.

The main limitation of the PSS tried in the laboratory or at the Mettur Thermal Plant is that the gains are based on a constant value of hunting frequency.

Although this PSS seems to provide much better results than a standard PSS, adapting the gains according to the changes in $P$ and $Q$, it will not, like all other PSS, be able to cope with local mode and interarea modes simultaneously or sequentially as the case may be.

Our efforts have, therefore, been directed towards identifying multiple frequencies of oscillation as and when they occur and using these frequencies in updating the gains and attempting to damp both the local mode and interarea mode as effectively as possible. Equations derived for a single machine infinite bus case have been modified for a multiple machine system (Ghosh 1991). The frequency identification is elaborated at some length in the section that follows.

\section{A novel multimachine adaptive PSS}

The basic principle involved in the cancellation of negative damping torque and ensuring dynamic stability has been outlined in $\$ 3.3 \mathrm{a}$. As indicated there, the analysis was carried out on an SMIB system and involved only the local mode of oscillation and hence, may not be very effective if a generator executes both local and interarea 
modes of oscillations. It is not uncommon that the generator initially responds to the higher frequency local mode which may be naturally damped after a few cycles but the interarea mode (which may be poorly damped because amortisseur windings are less effective at low frequencies) persists and needs to be damped.

In order to damp out different frequencies, it is necessary to estimate these frequencies first and then synthesize a PSS that can damp oscillations of multiple modes. Equation (11) providing expressions for $k_{\omega}$ and $k_{P}$ constants obtained for an SMIB also needs to be modified in order to represent a multimachine configuration.

\subsection{The h estimation procedure}

The popular method of spectral analysis, the FFT, is not suitable in the present case due mainly to the necessity of observing the process for a long time to obtain the required spectral resolution and the distortions introduced by spectral leakage into sidelobes. The modelling and parameter identification approach, which is a three-step process, has been therefore used.

Step 1: Selection of a discrete time domain model.

Step 2: Estimation of the parameters of the assumed model from available data samples.

Step 3: Obtaining the spectral estimates by substituting the estimated parameters into the theoretical PSD implied by the model.

Assuming a discrete time linear AR model, the system function in frequency domain can be written as,

$$
H_{d}(f)=1 /\left[1+\sum_{k=1}^{p} a_{k} \exp (-j 2 \pi f k T)\right],
$$

the PSD of an output sequence $\{x(n)\}$ is therefore,

$$
P_{d x}(f)=P_{d n}(f) /\left|\left[1+\sum_{k=1}^{p} a_{k} \exp (-j 2 \pi j k T)\right]\right|^{2},
$$

where $P_{d n}(f)$ is the PSD of the input sequence $\{n(n)\}$.

For discrete samples of a white noise,

$$
P_{d n}(f)=T R_{n}(0)=T \sigma_{n}^{2},
$$

where $T$ is the sampling time, and $\sigma_{n}^{2}=R_{n}(0)$ is the variance of white noise samples exciting the linear system. Hence,

$$
P_{d x}(f)=T \sigma_{n}^{2} /\left|\left[1+\sum_{k=1}^{p} a_{k} \exp (-j 2 \pi f k T)\right]\right|^{2} .
$$

The PSD can be determined if the model order $p$, the AR coefficients $\left\{a_{k}\right\}$ and the variance $\sigma_{n}^{2}$ of the white noise sequence are known. The AR model parameters are estimated by recursive computations using Burg's algorithm (Burg 1967) till the error power $\sigma_{n}^{2}$ does not change appreciably, indicating that the model is a sufficiently good approximation of the process.

For power systems applications, the model order $(p)$ and the number of data samples $(n)$ required for reliable $h$-estimation have to be carefully chosen. In addition, 
the spectrum of the signal is time variant and therefore only short data segments may be considered to be locally stationary. This requires that both the number of samples $(n)$ and the model order $(p)$ must be dynamically changed during the ' $h$ ' estimation process.

The values for $n$ and $p$ have been chosen from the a priori knowledge of the bandwidth of the signal spectrum which is of interest $(0.2$ to $3 \mathrm{~Hz})$ and are typical of most power systems. The lower end of the spectrum is associated with interarea modes and the higher end with intraplant modes. In between are the local modes, ranging from 0.8 to $1.6 \mathrm{~Hz}$. The signal to be analysed is, therefore, band limited by a suitable filter. The filter eliminates the steady-state drifts and reduces the computation time. Within this frequency range of interest, to obtain a reliable frequency identification algorithm, a sampling rate of about $10 \mathrm{~Hz}$ is necessary. The choice of model order is critical. If too large, spectral estimates exhibit spurious peaks, and if too low, an excessively smooth spectral estimate is obtained making identification of dominant modes difficult. A number of simulation and estimation studies were carried out with different model orders. Model orders in the range of $(n / 3) \leqslant p \leqslant(n / 2)$ were found to be suitable, which were also low enough to permit real-time computation.

Following the detection of a disturbance, the ' $h$ ' identifier is designed to gather and operate on the first 10 data samples. After two seconds, it operates on 20 samples, and after the third second, on 30 samples. However, from the end of the fourth second
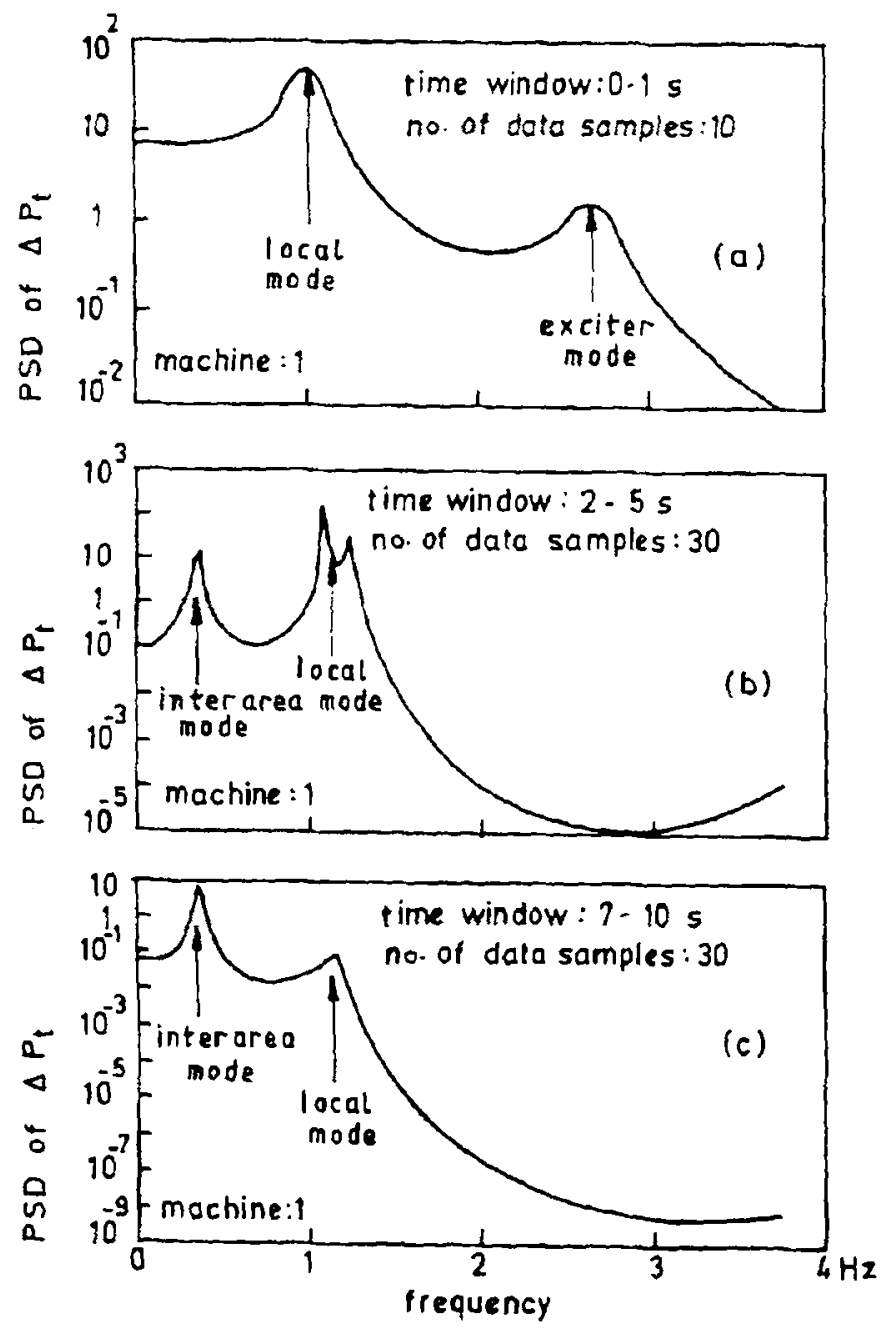

Figure 4. Identified dominant frequencies following a disturbance. (a) The local and the exciter modes; (b) interarea mode appears, local mode still dominant; (c) interarea mode becomes dominant, the local mode is damping out. 
rds, it operates on the last 30 data samples, discarding the earlier samples. This ig window' technique, with an overlap of 20 old and 10 fresh data samples it during the initial phase) ensures sufficient accuracy in detecting low frequency $s$, which need more data samples than high frequency modes at the same level uracy. Since the identifier works on recent data samples, it yields the changing al picture of the process. Typical $\Delta P$, spectra of one machine in a multimachine $n$, as obtained by the ' $h$ ' estimator at three different instances, are shown in 4. The dominant mode of oscillation in the initial period is about $1 \mathrm{~Hz}$ whereas sminant frequency at the later part is $0.3 \mathrm{~Hz}$.

itructure of the PSS

egative damping cancellation technique for the PSS design can now be extended nultimachine system. Supplementary excitation signals at each machine, $V_{\text {PSS, }}$, :he same form, i.e.,

$$
V_{\mathrm{PSS}}=k_{P} \Delta P+k_{\omega} \Delta \omega
$$

e gains of the pSs $k_{p}$ and $k_{\omega}$ are now determined by

$$
\begin{aligned}
& k_{p}=\left(B_{r}-h \omega_{0} D_{i}\right) /\left(h \omega_{0} C_{i}-A_{r}\right), \\
& k_{\omega}=\left(C_{r} k_{p}+D_{r}\right)+\left(A_{i} k_{p}+B_{i}\right) /\left(h \omega_{0}\right),
\end{aligned}
$$

${ }_{r}, A_{i}, B_{r}, B_{i}, C_{r}, C_{i}, C_{r}, D_{i}$, and $D_{r}$ are functions of

3 hunting frequency ' $h$ ',

$\approx$ equivalent line reactance ' $X_{e}$ ',

ıchine parameters, and

zoperating point $\left(V_{10}, P_{10}, Q_{10}\right)$ (see Ghosh 1991).

negative damping produced by multiple. modes are cancelled by identifying sst dominant frequency from the PSD every second and then synthesizing the ynal appropriate to the identified dominant frequency at that instant.

\section{'imulation results}

amic flux linkage multimachine model was used for the purpose of our studies. nfiguration is shown in figure 5 . Widely different machine ratings were chosen so lighlight the performance of the self-tuning PSS on small as well as very large nes. Each unit is represented by a tenth order dynamical model consisting of th-order synchronous generator, first-order AVR, first-order turbine and firstgovernor.

value of ' $h$ ' is not available to the PSS algorithm because of a minimum oneI observational delay. The machines are therefore operated with fixed-gain zers in the first second following a disturbance. The gains are 'tuned' as soon ' $h$ ' identifier results are available. Since $X_{e}$ is computed from steady-state values e computation is very simple, its value can be assumed to be available instansly (see Sen \& Ghosh 1991).

le 1a shows a very standard network and operating condition. All machines erating at near full load and $Z_{4}$ represents a typical $400 \mathrm{kV}, 500 \mathrm{kM}$ tie line. at buses 4 and 5 are largely met by local generation with very little power 


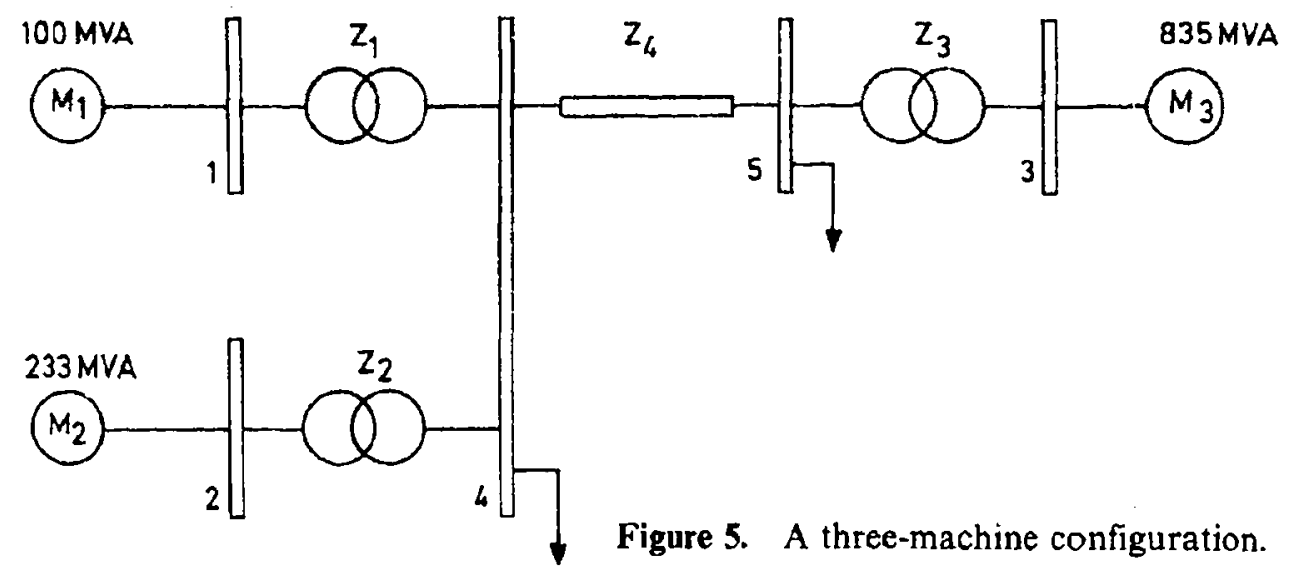

Table 1. System operating conditions (100 MVA base).

(a)

\begin{tabular}{lcc}
\hline $\begin{array}{l}\text { Active power } \\
P_{t} \text { (p.u.) }\end{array}$ & $\begin{array}{c}\text { Reactive power } \\
Q_{1} \text { (p.u.) }\end{array}$ & $\begin{array}{c}\text { Bus voltage } \\
V_{1} \text { (p.u.) }\end{array}$ \\
\hline$P_{1}=0.80$ & $Q_{1}=0.30$ & $V_{1}=1.02$ \\
$P_{2}=1.86$ & $Q_{2}=0.70$ & $V_{2}=1.03$ \\
$P_{3}=6.68$ & $Q_{3}=2.50$ & $V_{3}=1.04$ \\
$P_{4}=-2.50$ & $Q_{4}=-0.75$ & $V_{4}=1.00$ \\
$P_{5}=-6.84$ & $Q_{5}=-1.68$ & $V_{5}=1.00$ \\
\hline
\end{tabular}

(b)

\begin{tabular}{lcc}
\hline $\begin{array}{l}\text { Active power } \\
P_{t} \text { (p.u.) }\end{array}$ & $\begin{array}{c}\text { Reactive power } \\
Q_{t} \text { (p.u.) }\end{array}$ & $\begin{array}{c}\text { Bus voltage } \\
V_{t} \text { (p.u.) }\end{array}$ \\
\hline$P_{1}=0.80$ & $Q_{1}=-0.15$ & $V_{1}=0.98$ \\
$P_{2}=1.86$ & $Q_{2}=0.70$ & $V_{2}=1.03$ \\
$P_{3}=6.68$ & $Q_{3}=-1.25$ & $V_{3}=0.97$ \\
$P_{4}=-4.00$ & $Q_{4}=0.01$ & $V_{4}=1.00$ \\
$P_{5}=-5.31$ & $Q_{5}=-2.17$ & $V_{5}=1.00$ \\
\hline
\end{tabular}

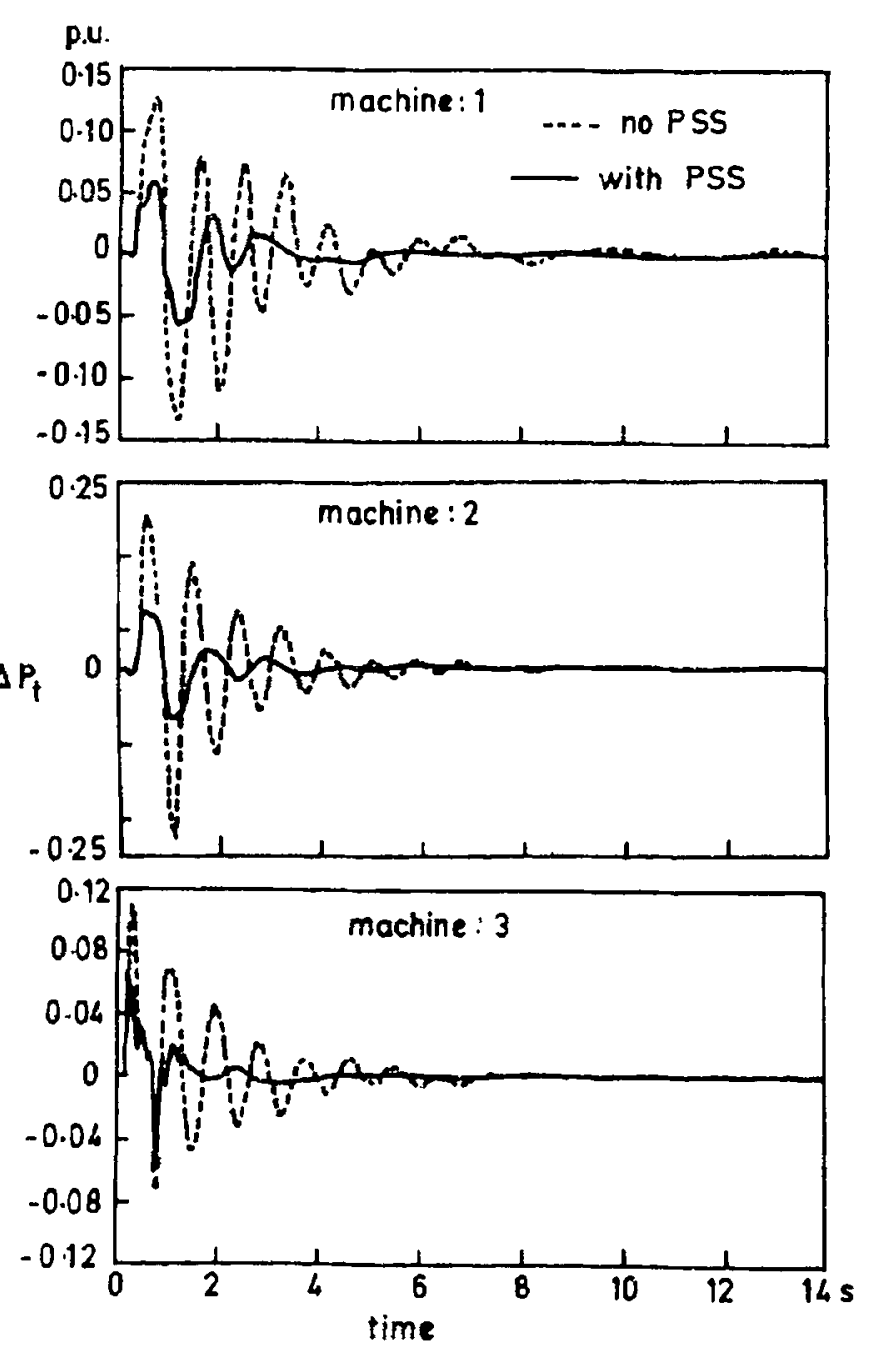

Figure 6. $\Delta P$, response (see table 1a) 
flow on the tie line. The $\Delta P_{t}$ responses of the system following a small perturbation are shown in figure 6.

Table $1 \mathrm{~b}$ relates to a rather difficult network condition. Both machines 1 and 3 are operating at near full loads with leading power factors. The tie is a $1000 \mathrm{kM}$, $400 \mathrm{kV}$ line. Active power loadings at buses 4 and 5 are comparable with bulk power flowing through the transmission line. Reactive powers are also imported at these buses to maintain the load voltages. The $\Delta P$, responses without the stabilizer clearly show the oscillatory nature of the weak system. The proposed PSS, however, has been able to stabilize the system to a considerable extent as shown in figure 7.

It is clear from figures $6 \& 7$, that the PSS does not play a very significant role in system condition - a, when natural damping is sufficiently large and the AVR does not introduce large negative damping for the stabilizer to cancel. On the other hand, for system condition - $b$, the system is highly oscillatory due to large negative damping torque generated by the AVR. The PSS cancels this negative torque and the system is well damped. In other words, the PSS seems to be effective when required.
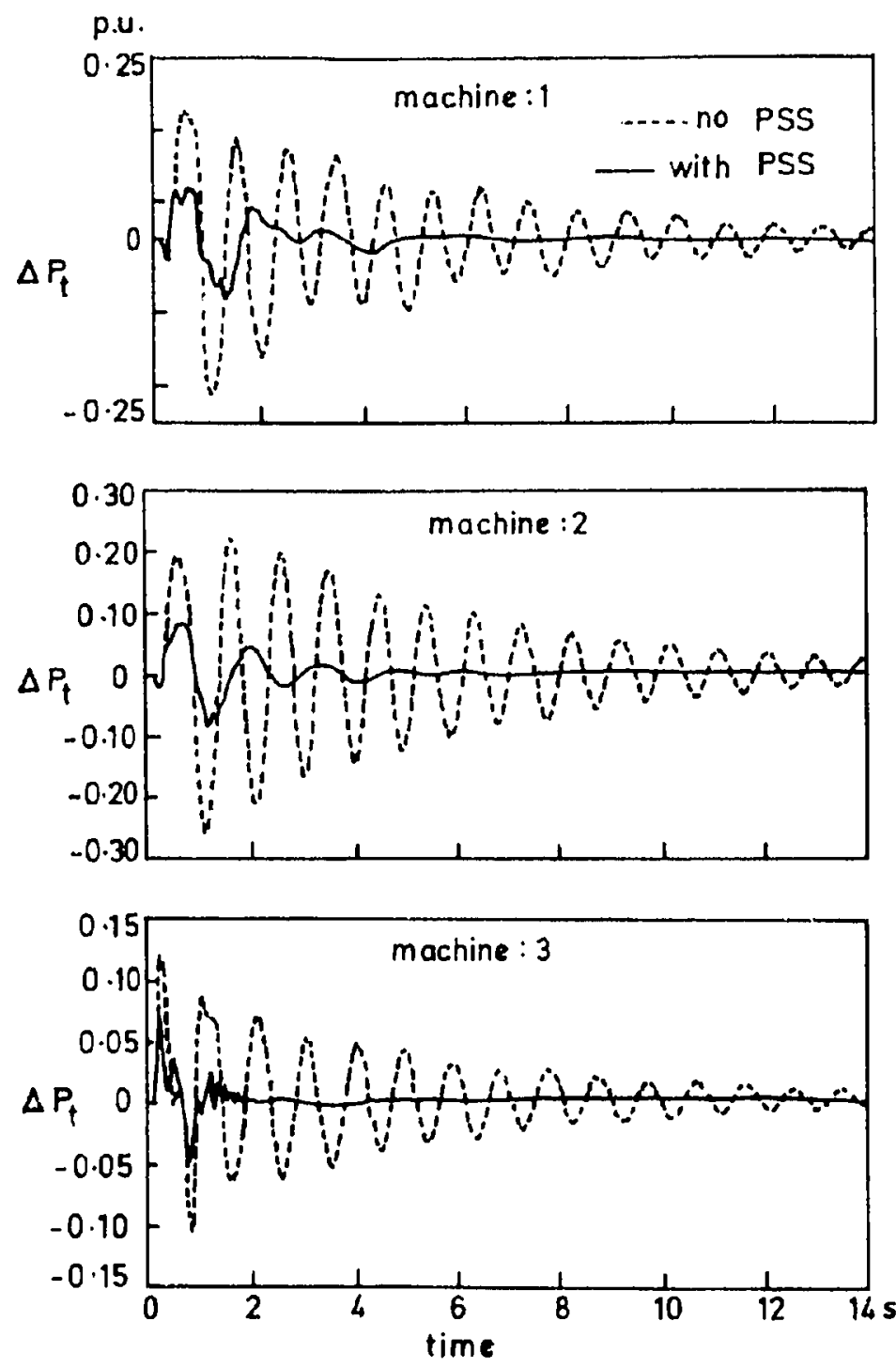

Figure 7. $\Delta P$, response (sce table 1a). 


\section{Conclusions}

The main factors causing low frequency oscillations in a synchronous generator have been identified and, having identified the causes, an attempt is made to cancel their effects to ensure stability. This enables the design of a stabilizer whose gains change with operating and system conditions. The approach is distinctly different from other attempts at designing adaptive stabilizers. The effectiveness of this new PSS was earlier established for local modes of oscillations. The PSS now modified for multimachine system, making use of the dominant frequency as obtained from the ' $h$ ' identification procedure in real-time, appears to be effective in damping out the multiple modes of oscillations.

\section{References}

Burg J P 1967 Maximum entropy spectral analysis. Proc. 37th Meeting (Oklahama: Soc. Explor. Geophys.)

Byerly R T, Bennon R J, Sherman D E 1982 Eigenvalue analysis of synchronizing power flow oscillations in large electric power systems. IEEE Trans. Power Appar. Syst. PAS-101: $235-245$

deMello F B, Concordia C 1969 Concepts of synchronous machine stability as affected by excitation control. IEEE Trans. Power Appar. Syst. PAS-88: 316-329

Farmer R G, Agrawal B L 1983 State-of-the-art techniques for power system stabilizer tuning. IEEE Trans. Power Appar. Syst. PAS-102: 699-709

Ghosh F 1991 On multimode stabilization of interconnected power systems. M Sc thesis, Indian Institute of Science, Bangalore

Gibson C M 1988 Programme of system proving tests and operational experience. Proc. Inst. Electr. Eng. C135: 255-260

Grund C E 1978 Power system model identification from large scale simulations using model reference adaptive control. IEEE Trans. Power Appar. Syst. PAS-97: 780-788

Hanson O W, Goodwin C J, Dandeno P L 1968 Influence of excitation and speed control parameters in stabilizing intersystem oscillations. IEEE Trans. Power Appar. Syst. PAS-87: $276-282$

Kanniah J, Malik O P, Hope G S 1984 Excitation control of synchronous generators using adaptive regulators I \& II. IEEE Trans. Power Appar. Syst. PAS-103: 897-910

Kron G 1952 A new theory of hunting. Trans. AIEE 71: 859

Kundur P, Klein M, Rogers G J, Zywno M S 1989 Application of power system stabilizers for enhancement of overall system stability. IEEE Trans. Power Syst. 4: 614-626

Larsen E V, Swann D A 1981 Applying power system stabilizers. I, II, \& III. IEEE Trans. Power Appar. Syst. PAS-100: 3017-3045

Nambiar T N P 1984 On dynamic stability and power system stabilizers in synchronous generators. $\mathrm{Ph} \mathrm{D}$ thesis, Indian Institute of Science, Bangalore

Narahari N G 1982 Control techniques to improve dynamic stability of synchronous generators. $\mathrm{Ph} \mathrm{D}$ thesis, Indian Institute of Science, Bangalore

Sen I, Ghosh $F 1991$ Identification of hunting frequency and equivalent transmission line impedance in a multimachine system for stability applications. Proc. Int. Conf. Advances in Power System Control, Operation \& Management (Hong Kong: Inst. Electr. Eng.)pp. 81-85

Sen Gupta D P, Lynn J W 1980 Electrical machine dynamics (New York, London: MacMillan)

Sen Gupta D P, Narahari N G, Boyd I, Hogg B W 1985 An adaptive power system stabilizer which cancels the negative damping torque of a synchronous generator. Proc. Inst. Electr. Eng. 132: 109-117

Sen Gupta D P, Narahari N G, Lynn J W 1977 Damping and synchronizing torque contributed by a voltage regulator to a synchronous generator. A quantitative assessment. Proc. Inst. Electr. Eng. 124: 702-708

Wu Q H, Hogg B W 1990 Self-tuning control for turbogenerators in multimachine power systems. Proc. Inst. Electr. Eng. 137: 146-158 


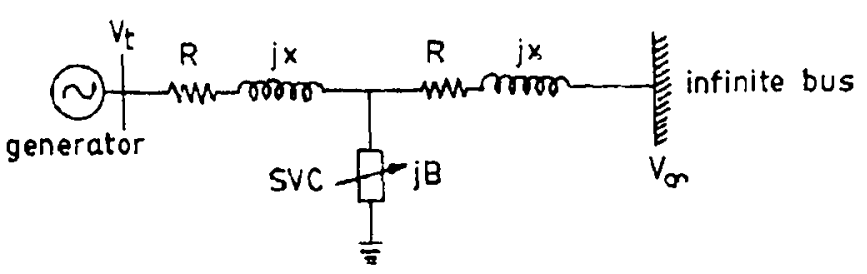

Figure 1. Single machine infinite bus system with SVC.

Alternately, some kind of adaptation mechanism for stabilizers should be used. Robustness analysis can also be used to choose the input signals for PSS and SVC stabilizers. This robustness aspect is considered in Larsen \& Swann (1981), Padiyar et al (1986), and Madhu \& Prabhu (1989, pp. 63-7, 1990). Among adaptation schemes, gain scheduling is the simplest, most effective and easiest to implement (Sen Gupta et al 1985; Bandyopadhyay \& Prabhu 1988; Madhu \& Prabhu 1989, pp. 63-7; Sen Gupta 1989).

There is an additional aspect that should be considered in stabilizer design. It is the possibility of controller failures. Though this is expected to be a rare event in practice, it cannot be ruled out. Stabilizer design should ensure satisfactory damping even under conditions of failure of some stabilizers. This paper considers this problem for a power system with a PSS and an SVC stabilizer. Under normal conditions, both stabilizers are in operation. Under abnormal conditions, only one stabilizer is in operation. The design requirement is that the system operate satisfactorily under these conditions in terms of a stability robustness criterion. Following Minto (1985), this requirement may be termed reliable decentralized stabilization. The design developed here uses the robustness criterion of Madhu \& Prabhu (1989, pp. 63-7).

The rest of the paper is organised as follows. Section 2 gives the power system considered and its model. Section 3 defines the control problem and outlines the computer-aided design (CAD) methodology used. Section 4 gives the results obtained for the particular problem considered. Section 5 gives the conclusions.

\section{Power system considered and its model}

The power system considered, consists of a synchronous generator connected to an infinite bus through a transmission line (figure 1). The generator has a PSS in its AVR system. There is an SVC with a stabilizer at the midpoint of the transmission line. As is usual for dynamic stability studies, the generator damper windings and saturation, and turbine-governor dynamics are neglected. Generator stator and external network are assumed to be in a quasi-steady state. A T-model for the transmission line is assumed to be sufficient and the line charging susceptance is clubbed with SVC susceptance.

A fast-acting solid state single time constant IEEE Type 1S excitation system (IEEE Committee Report 1968) is considered for the generator. SVC voltage regulator is also a single time constant system. Linearized dynamical equations of the system are derived at an operating point along the lines of the Hefron Philips model (Anderson \& Fouad 1977). See appendix A for details. The linear model is of fifth order with two inputs, namely, the stabilizing signals for the PSS and the SVC stabilizer. It has the form

state equation:

$$
\dot{\mathbf{x}}=A \mathbf{x}+\mathbf{b}_{1} u+\mathbf{b}_{2} u_{2}
$$




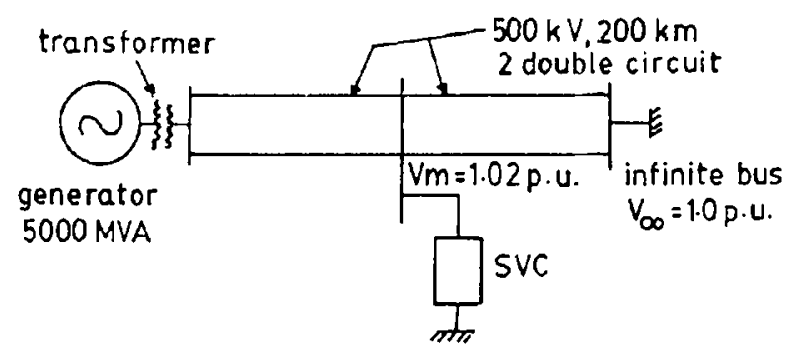

Figure 2. Example system.

where

$$
\mathbf{x}=\left[\Delta E_{q}^{\prime}, \Delta \omega, \Delta \delta, \Delta E_{F D}, \Delta B\right]^{T}
$$

The state variables in (2) are expressed in the standard notation (Anderson \& Fouad 1977). $\Delta B$ denotes incremental change in svc susceptance. Furthermore, $u_{1}=\Delta V_{s s}$ and $u_{2}=\Delta V_{s e}$ are the stabilizing signal outputs of PSS and SVC stabilizers, respectively.

The output variables of the system are defined to be those that are used as input to the stabilizers. For PSS the input signals considered are generator incremental rotor speed and active power. SVC stabilizer input signals are incremental midline active power and SVC bus angular frequency. Corresponding to each of these signals, there is an output equation of the system.

The output equation corresponding to each of the PSS inputs has the form

$$
y_{1}=C_{1} \mathbf{x} .
$$

For the case of SVC stabilizer, the corresponding output equations have the form

$$
y_{2}=C_{2} \mathbf{x}+d_{2} u_{2} \text {. }
$$

The matrices $C_{1}, C_{2}$ and the scalar $d_{2}$ for the output variables considered are given in appendix $A$.

The particular example system chosen for detailed study is the same as that of Kinoshita (1979) except for excitation system data (figure 2) and generator inertia control, $H$. Generator capacity is $5000 \mathrm{MVA}$. This generator is an equivalent representation for all the generators at the generating station. Power is transmitted over $200 \mathrm{~km}$ on two $500 \mathrm{kV}$ double circuit lines (4 lines in parallel, in all). SVC is installed at the midpoint of the transmission line. Power system data are given in appendix B. The infinite bus voltage is 1 p.u. on a $500 \mathrm{kV}$ base. It is decided to regulate SVC bus voltage at $1.02 \mathrm{p}$.u. The design point for design of stabilizers is considered to be generator operating at rated capacity and 0.9 lagging p.f.

A load flow analysis is done to determine the generator terminal conditions and SvC bus voltage angle. These results are utilized to determine the parameters in system equations (1), (3) and (4). The system poles (eigenvalues of matrix $A$ in (1)) corresponding to this condition are $-148.54,-98.85,-1.987,-0.1116 \pm j 12.79$. This indicates unsatisfactory damping of the electromechanical mode.

\section{Control problem and CAD methodology used}

\subsection{Structure of PSS and SVC stabilizers}

Following the industry practice and neglecting filter and washout stages, PSS and svC stabilizers are considered to have second-order transfer functions as given below. 


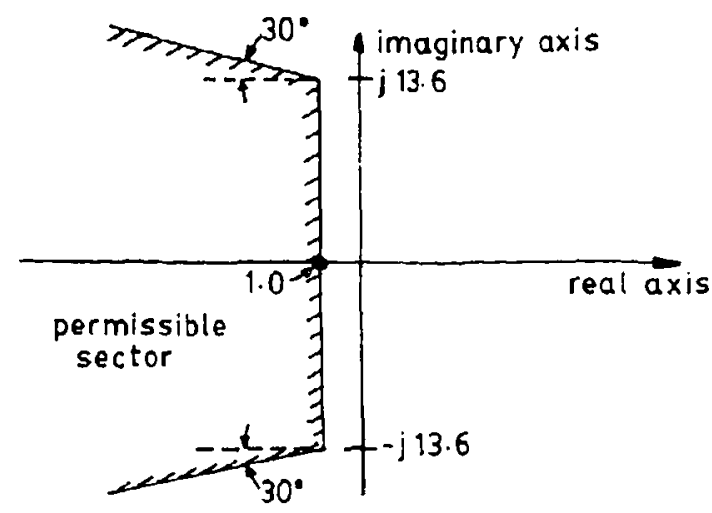

Figure 3. Sector showing permissible locations of closed loop eigenvalues.

PSS transfer function

$$
F_{p}(s)=\left(\theta_{o p} s^{2}+\theta_{1 p} s+\theta_{2 p}\right) /\left(s^{2}+\gamma_{1 p} s+\gamma_{2 p}\right) .
$$

SVC stabilizer

$$
F_{c}(s)=\left(\theta_{o c} s^{2}+\theta_{1 c} s+\theta_{2 c}\right) /\left(s^{2}+\gamma_{1 c} s+\gamma_{2 c}\right)
$$

\subsection{Sector criterion for pole assignment and robustness}

Following Madhu \& Prabhu (1989, pp. 63-7, 1990), the sector in the complex plane shown in figure 3 is considered, for PSS and SVC stabilizer design by pole assignment, as well as for assessing the robustness of the design. This sector is chosen on the basis of the location of the poles of the system without stabilizers, and the minimum damping of the electromechanical mode deemed satisfactory (Sharma 1991). The stabilizers are designed for a particular operating point. The design is said to be robust with respect to a given variation in $P$ and $Q$ if the system poles remain in the sector for these variations. The region of robustness in the $P-Q$ plarfe is the largest set of operating points in the plane for which the design remains robust.

\subsection{Control problem}

The control problem is to design a PSS and an SVC stabilizer, each giving a reasonably large robustness region when operating alone, and with the region, when both operate together, being at least equal to the union of the two individual robustness regions.

It is not known a priori whether such a design exists. There is no theory at present which answers this question. However, the design methodology developed tries to find a solution assuming that one exists.

It is to be noted that this design is a reliable, decentralized controller design in the presence of a possibility of controller failure.

\subsection{Controller design}

The design point chosen is generator at full load with 0.9 lagging p.f. This is done because (i) the system is expected to operate under this condition most of the time, and (ii) dynamic stability is known to worsen under heavily loaded conditions. 
Each stabilizer is of second order. The power system with AVR and SVC voltage regulator is a fifth-order single-input single-output system. Under these conditions, only 3 poles can be arbitrarily assigned (Munro \& Hirbod 1979), using only one stabilizer. Each stabilizer is designed for a chosen input to it, assuming the other to be absent, by partial pole assignment (Munro \& Hirbod 1979), following the procedure laid down in Madhu \& Prabhu (1989, pp. 63-7, 1990). An important design consideration is the choice of the 3 poles to be assigned and their closed loop locations in the sector chosen. Electromechanical and exciter modes are chosen for this assignment. The choice of locations involves experimentation, since inappropriate values may require large loop gains. After the assignment, the locations of the unassigned closed loop eigenvalues are checked to ensure that they lie within the sector chosen. See Sharma (1991) for details.

\subsection{Coordination of PSS and SVC stabilizer designs}

Following the procedure given above, several PSS are designed for different locations of assigned eigenvalues. The corresponding eigenvalues in the different designs differ from each other by small amounts. In a similar way, several sets of SVC stabilizers are also designed. For each design, the corresponding robustness region is determined. Then various combinations of PSS and SVC stabilizers are considered, and for each combination of stabilizers working together, the corresponding robustness region is determined. A combination is then chosen which gives a robustness region containing in it the robustness regions corresponding to the PSS and SVC stabilizer in the combination acting alone. Though this may appear to involve a large computational effort, we have found that it is sufficient to consider a fairly small number of individual stabilizer designs.

\section{Numerical results}

Two types of PSS, namely, speed $(\Delta \omega)$ input PSS and power $\left(\Delta P_{e}\right)$ input PSS are designed. Similarly, two types of SVC stabilizer, one with bus frequency $\left(\Delta \omega_{s}\right)$ input and the other with midline power $\left(\Delta P_{m}\right)$ input are designed. In all 12 individual stabilizer designs are made, three for each type of input. Design of washout stages and signal conditioning filters is not a part of this design.

Table 1 gives the assigned eigenvalues and PSS transfer function for $\Delta \omega$ and $\Delta P_{e}$

Table 1. PSS designs.

\begin{tabular}{lc}
\hline & Power $\left(\Delta P_{e}\right)$ input PSS \\
\hline Eigenvalues assigned & PSs transfer functions, $F_{s}(s)$ \\
\hline$-1.54 \pm j 13 \cdot 2,-2 \cdot 5$ & $(-3)\left(0.098 s^{2}+3 \cdot 4 s+23 \cdot 6\right) /\left(s^{2}+24 s+80\right)$ \\
$-1 \cdot 5 \pm j 13 \cdot 0,-2 \cdot 2$ & $(-3)\left(0 \cdot 114 s^{2}+3 \cdot 2 s+16 \cdot 6\right) /\left(s^{2}+24 s+80\right)$ \\
$-1 \cdot 5 \pm j 12 \cdot 8,-2 \cdot 0$ & $(-3)\left(0.08 s^{2}+2 \cdot 89 s+6 \cdot 3\right) /\left(s^{2}+24 s+80\right)$ \\
& Speed $(\Delta \omega)$ input PSs \\
$-2 \cdot 5 \pm j 13 \cdot 2,-2 \cdot 0$ & $(-1)\left(-0.138 s^{2}-0.62 s-0.51\right) /\left(s^{2}+30 s+200\right)$ \\
$-2 \cdot 0 \pm j 13 \cdot 0,-2 \cdot 0$ & $(-1)\left(-0.113 s^{2}-0.426 s-0.22\right) /\left(s^{2}+30 s+200\right)$ \\
$-1 \cdot 5 \pm j 13 \cdot 6,-2 \cdot 0$ & $(-1)\left(-0.092 s^{2}-0.81 s-1 \cdot 07\right) /\left(s^{2}+30 s+200\right)$ \\
\hline
\end{tabular}


Table 2. SVC stabilizer designs.

\begin{tabular}{|c|c|}
\hline \multicolumn{2}{|c|}{ Midline power $\left(\Delta P_{m}\right)$ input svC stabilizer } \\
\hline Eigenvalues assigned & SvC stabilizer transfer function, $F_{c}(s)$ \\
\hline $\begin{array}{l}-1 \cdot 5 \pm j 13.0,-2 \cdot 2 \\
-1.54 \pm j 13 \cdot 4,-2.5 \\
-1.5 \pm j 12 \cdot 8,-2 \cdot 0\end{array}$ & $\begin{array}{l}(-3)\left(-0.253 s^{2}-4.4 s-10.51\right) /\left(s^{2}+24 s+80\right) \\
(-3)\left(-0.213 s^{2}-5 \cdot 136 s-15.41\right) /\left(s^{2}+24 s+80\right) \\
(-3)\left(-0.271 s^{2}-4.086 s-7.3\right) /\left(s^{2}+24 s+80\right)\end{array}$ \\
\hline & svC bus frequency $\left(\Delta \omega_{s}\right)$ input svc stabilizer \\
\hline $\begin{array}{l}-1.58 \pm j 10.93,-2 \cdot 2 \\
-1.5 \pm j 10.89,-2 \cdot 0 \\
-1.54 \pm j 13 \cdot 2,-2.5\end{array}$ & $\begin{array}{l}(-1)\left(-0.031 s^{2}-2.7 s-6.73\right) /\left(s^{2}+24 s+80\right) \\
(-1)\left(-0.027 s^{2}-2.67 s-5 \cdot 31\right) /\left(s^{2}+24 s+80\right) \\
(-1)\left(-0.046 s^{2}-2.8 s-8.4\right) /\left(s^{2}+24 s+80\right)\end{array}$ \\
\hline
\end{tabular}

input PSS. Table 2 gives the corresponding quantities for $\Delta \omega_{s}$ and $\Delta P_{m}$ input sV stabilizers.

Using the approach outlined in $\S 3.5$ we now choose particular combinations $\mathrm{c}$ PSS and SVC stabilizer designs such that when these two stabilizers operate simul taneously, the corresponding robustness region contains the regions for individua stabilizers operating alone.

We have determined the best design for each type of PSS and SVC stabilizer inputs In all there are four input combinations, namely,

(i) $\Delta P_{e}$ input PSS with $\Delta P_{m}$ input SVC stabilizer,

(ii) $\Delta \omega$ input PSS with $\Delta \omega_{s}$ input SVC stabilizer,

(iii) $\Delta P_{e}$ input PSS with $\Delta \omega_{s}$ input SVC stabilizer,

(iv) $\Delta \omega$ input PSS with $\Delta P_{m}$ input SVC stabilizer.

Figures 4 to 7 give the robustness regions for the coordinated PSS-SVC stabilize: designs for the cases (i) to (iv), respectively. These figures also give the transfer function: of the individual stabilizers.

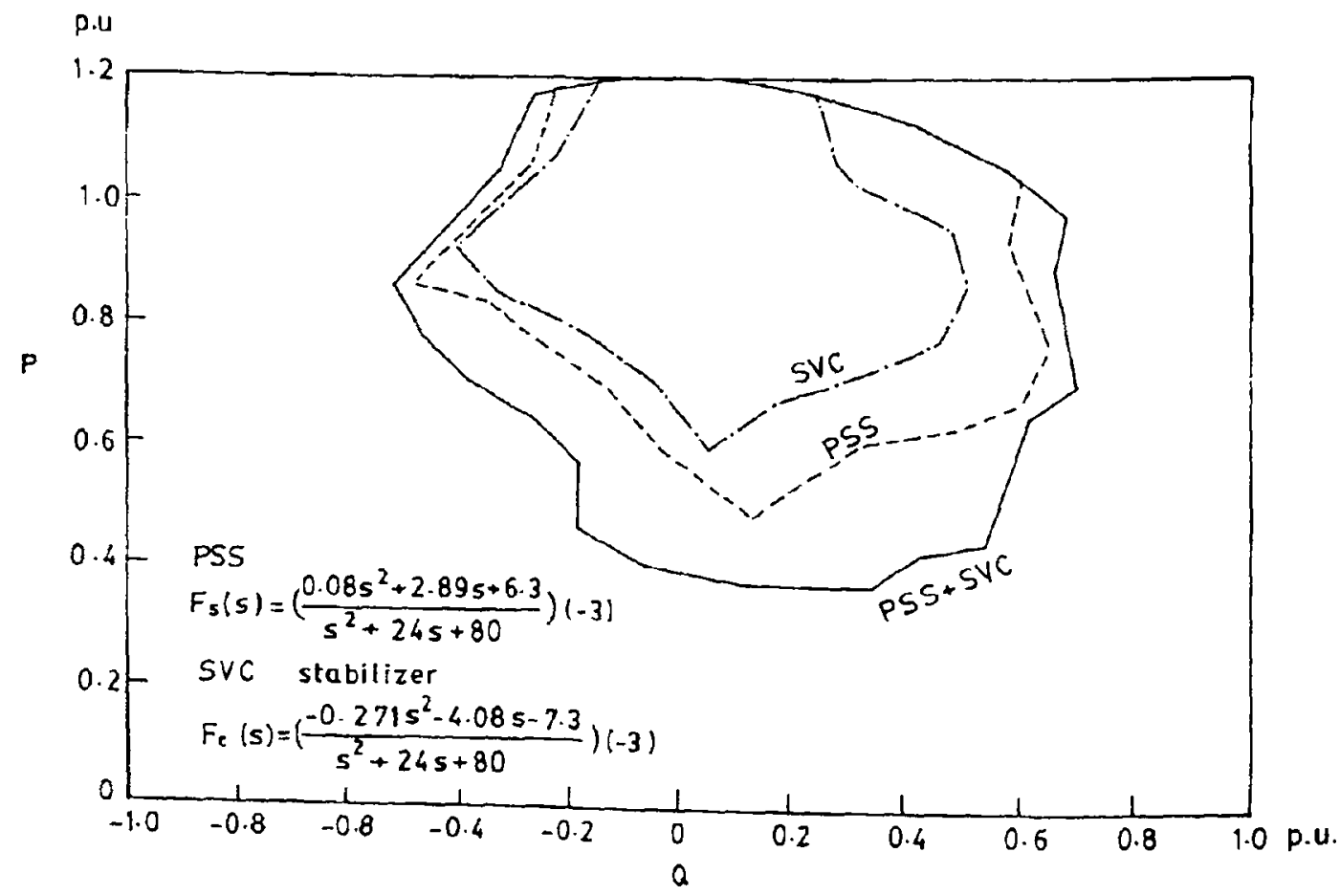

Figure 4. Regions of robustness for $\Delta P_{e}$ input PSS and $\Delta P_{m}$ input SVC stabilizer. 


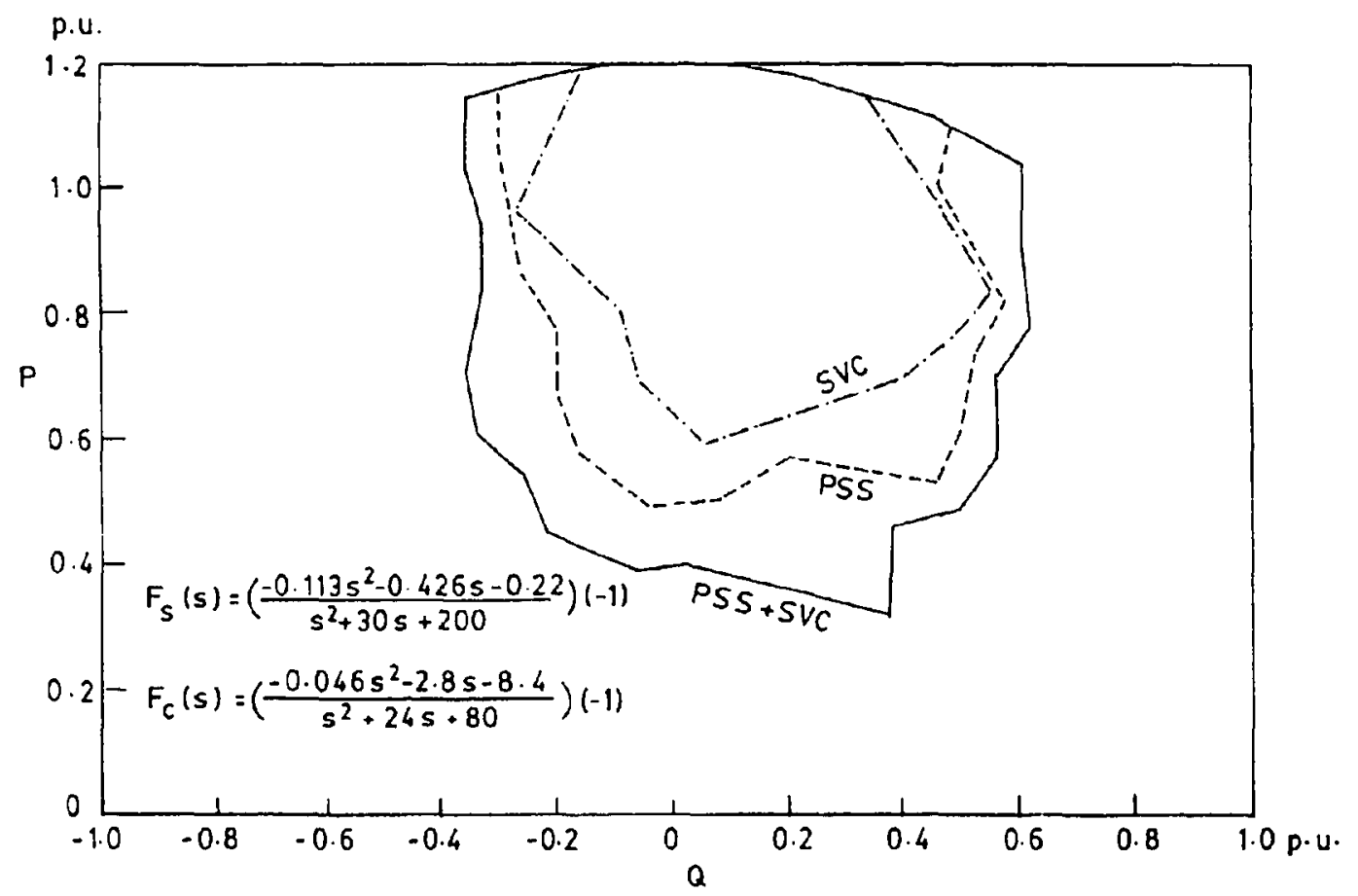

Figure 5. Regions of robustness for $\Delta \omega$ input PSS and $\Delta \omega_{s}$ input svc stabilizer.

\subsection{Remarks}

(1) In finding the robustness regions, we have not investigated the region beyond $1 \cdot 2$ p.u. generator loading. The regions obtained are thus bounded by the line corresponding to this loading.

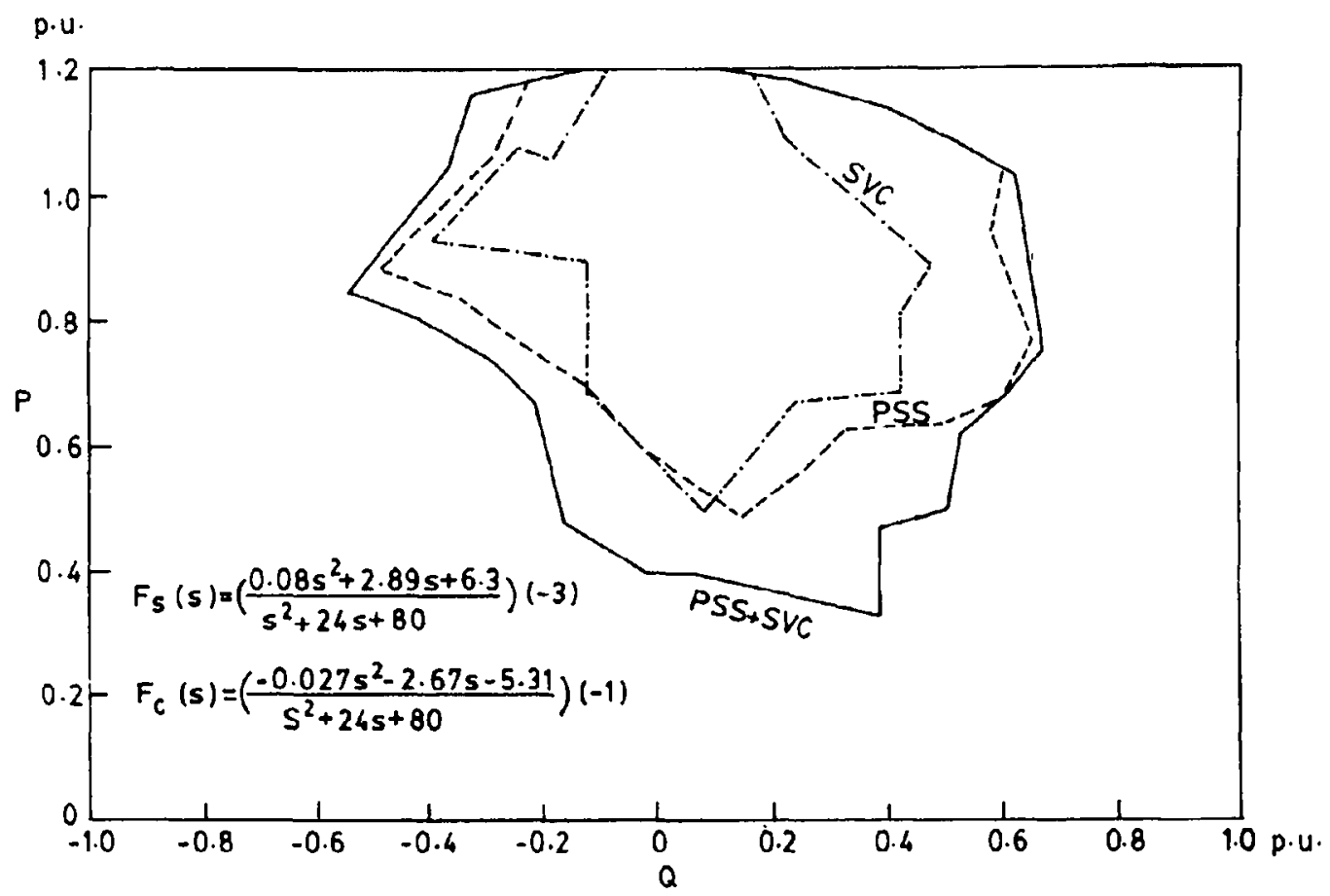

Figure 6. Regions of robustness for $\Delta P_{e}$ input PSS and $\Delta \omega_{s}$ input SVC stabilizer. 


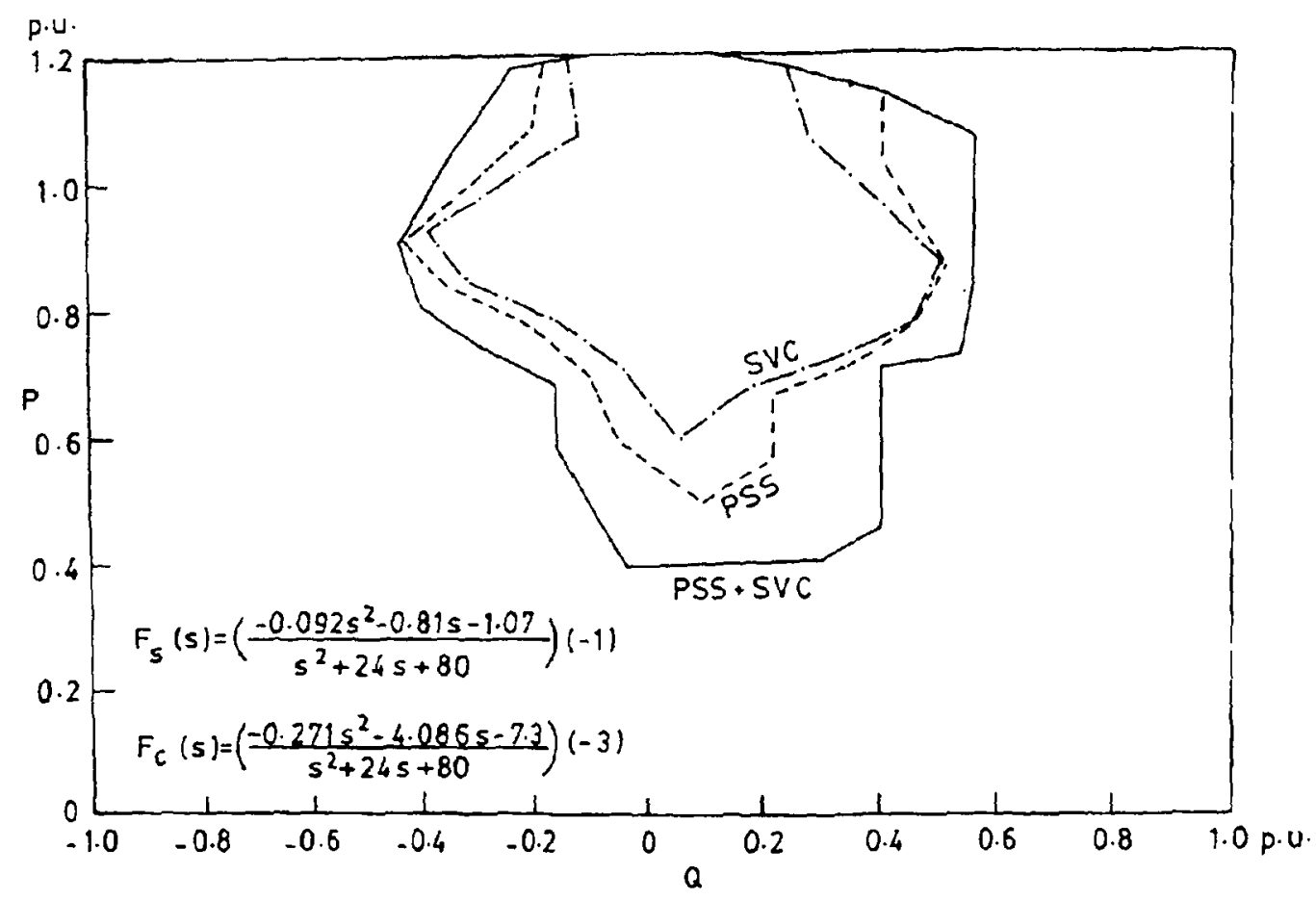

Figure 7. Regions of robustness for $\Delta \omega$ input PSS and $\Delta P_{m}$ input SVC stabilizer.

(2) In all cases it is observed that the robustness region for a PSS is larger than that for an SVC stabilizer. This indicates that a PSS is more effective in dynamic stability improvement than an SVC stabilizer for the problem considered.

(3) The robustness regions for stabilizer combinations other than those in figures 1 to 4 are given in Sharma (1991) and are not repeated here. These results clearly indicate the need for proper choice of individual stabilizers.

(4) A simple rule seems to emerge for a well-coordinated design of PSS and SVC stabilizers for the problem considered. That is, choose individual stabilizers so that their individual robustness regions overlap by a large amount. Then, if they are operated together, the corresponding robustness region will also overlap the individual regions by a large amount, thus giving a satisfactory design. This is truc whatever may be the inputs selected for the individual stabilizers.

\section{Conclusions}

This paper has presented a new design concept for coordinated design of PSS and SVC stabilizers for power systems in order that good performance of the system may be obtained over a wide range of operating conditions, judged in terms of a robustness criterion, when both stabilizers are operating and when any one of them fails. Theoretical results are lacking in this area. However, a computer-aided design (CAD) approach has been presented for obtaining satisfactory design. The procedure has been illustrated by use of an example and relevant numerical results have been presented. An empirical rule has emerged for obtaining a coordinated PSS and SVC stabilizer design. 


\section{Appendix A. Power system model}

\section{Generator model}

A third-order generator model is used. The equations and notations are standard; see, for example, Anderson \& Fouad (1977). The equations are given below.

where

$$
\begin{aligned}
p E_{q}^{\prime} & =-\left(E_{q}^{\prime} / T_{d o}^{\prime}\right)+\left(E_{F D} / T_{d o}^{\prime}\right)+\left(x_{d}-x_{d}^{\prime}\right) I_{d}, \\
p \omega & =(1 / M)\left(T_{m}-T_{e}\right)-\left(K_{D} / M\right)\left(\omega-\omega_{0}\right), \\
p \delta & =\omega-\omega_{0}
\end{aligned}
$$

$$
\left.\begin{array}{l}
M=2 H / \omega_{0}, \\
T_{e}=V_{d} I_{d}+V_{q} I_{q}, \\
V_{d}=-x_{q} I_{q}, \\
V_{q}=x_{d}^{\prime} I_{d}+E_{q}^{\prime} .
\end{array}\right\}
$$

All quantities are in p.u. except $\delta$ and $\omega$ which are in radians and radians/second, respectively.

\section{Excitation system model}

A fast-acting solid state single time constant IEEE Type 1S excitation system is considered. Figure A1 gives a block diagram of the system. The differential equation for the system is

where

$$
p E_{F D}=-\left(E_{F D} / T_{A}\right)+\left(K_{A} / T_{A}\right)\left(V_{\text {ref }}-V_{s}-V_{s s}\right),
$$

$$
V_{t}=\left(V_{d}^{2}+V_{q}^{2}\right)^{1 / 2}
$$

SVC model

A single time constant SVC voltage controller is considered. Figure A2 gives a block diagram of the controller. The differential equation for the system is

$$
p B=-\left(B / T_{B}\right)+\left(K_{B} / T_{B}\right)\left(V_{\text {mref }}-V_{m}+V_{s e}\right)
$$

\section{Linearized model}

The linearized state space model at an operating point is derived by linearizing equations (A1)-(A6). The nonstate variables $\Delta I_{d}, \Delta T_{e}, \Delta V_{t}$ and $\Delta V_{m}$ are represented

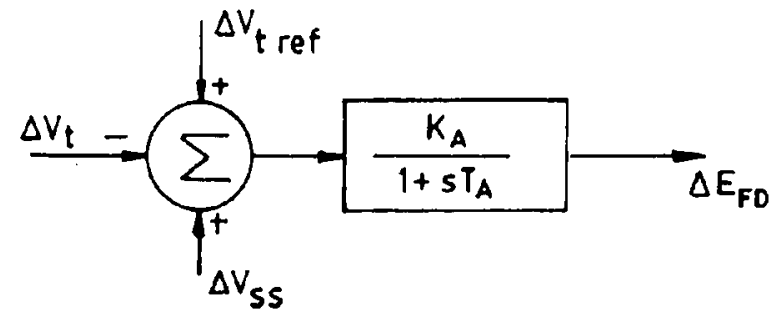

Figure A1. AVR system. 


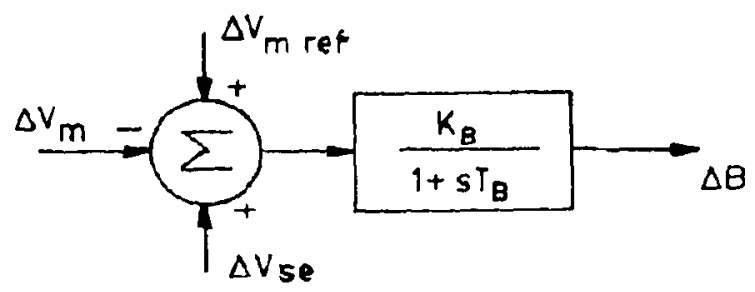

Figure A2. SvC voltage controller.

in terms of the state variables $\Delta E_{a}^{\prime}, \Delta \omega, \Delta \delta, \Delta E_{F D}$ and $\Delta B$. The expressions are complex and lengthy and involve the various system parameters (operating conditions, network, generator, exciter and SVC parameters). They are given in detail in Sharma (1991).

\section{Output equations}

The output equations for the various signals used for PSS and SVC stabilizers have the form given below. See (3), (4) in the text.

Velocity input PSS

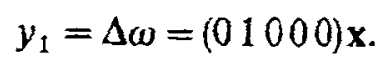

Power input PSS

$$
y_{1}=\Delta P_{e}=\left[C_{1} O C_{2} O C_{3}\right] \mathbf{x} .
$$

Midline power input SVC stabilizer

$$
y_{2}=\Delta P_{m}=\left[C_{4} 0 C_{5} 0 C_{6}\right] \mathbf{x} .
$$

Bus angular frequency input SVC stabilizer

$$
y_{2}=\Delta \omega_{s}=\left[C_{7} C_{8} C_{9} C_{10} C_{11}\right] \mathbf{x}+C_{12} \Delta V_{s e}
$$

The expressions for the constants $C_{1}-C_{12}$ are complex and involve the various system parameters. They are given in detail in Sharma (1991)

\section{Appendix B. Power system data}

Most of the data are as in Kinoshita (1979). All quantities are expressed in p.u. on a $500 \mathrm{kV}, 5000 \mathrm{MVA}$ base unless otherwise specified. These values are the generator line voltage and power ratings, respectively.

Generator

$$
\begin{aligned}
x_{d} & =1.7, & x_{q}=1.44, & x_{d}^{\prime}=0.345, \\
T_{d o}^{\prime} & =6.4, & H=1.4 \mathrm{~s}, & \omega_{0}=314 \mathrm{rad} / \mathrm{s} .
\end{aligned}
$$

Excitation system

$$
K_{A}=50, \quad T_{A}=0.01 \mathrm{~s} .
$$


SVC voltage controlled

$$
K_{B}=20, \quad T_{B}=0.02 \mathrm{~s} .
$$

Network parameters

(i) Step-up transformer, $x_{t}$ neglected.

(ii) Transmission line.

Two double circuit lines.

Line constants for each circuit for $100 \mathrm{~km}$ length:

$r_{\text {line }}=0.0135$

$x_{\text {line }}=0.452$

$B_{\text {line }}=0.02294$

Infinite bus voltage: $1.0 \mathrm{~L} 0^{\circ}$

$K_{D}=0$.

\section{List of symbols}

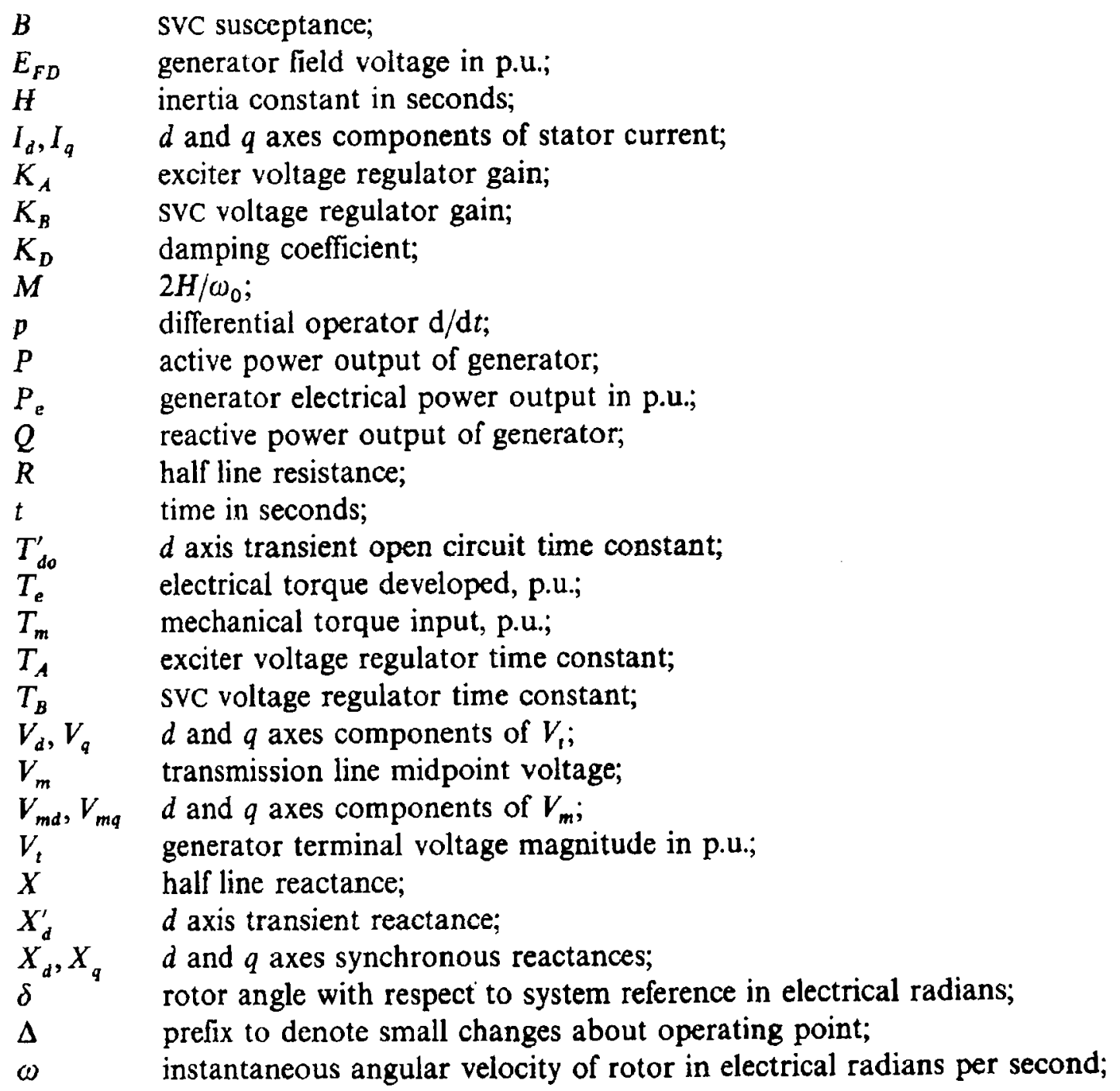


$\omega_{0} \quad$ synchronous angular velocity in electrical radians per second;

$\omega_{s} \quad$ angular frequency of syc bus in radians per second;

o subscript to denote the value of the operating point;

$T \quad$ superscript denoting transpose operation.

\section{References}

Anderson P M, Fouad A A 1977 Power system control and stability (Ames, IA: Iowa State University Press)

Bandyopadhyay G, Prabhu S S 1988 A new approach to adaptive power system stabilizers. Electr. Mach. Power Syst. 14: 111-125

IEEE Committee Report 1968 Computer representation of excitation systems. IEEE Trans. Power Appar. Syst. PAS-87: 1460-1464

Kinoshita $H 1979$ Improvement of power system dynamic stability by static shunt var system. Electr. Eng. Jpn. 99: 81-89

Larson E V, Swann D A 1981 Applying power system stabilizers. IEEE Trans. Power Appar. Syst. PAS-100: 3017-3046

Madhu G N, Prabhu S S 1989 Computer-aided design of a robust composite adaptive power system stabilizer. Proc. NACONECS-89 (New Delhi: Tata McGraw Hill)

Madhu G N, Prabhu S S 1990 A new method for evaluating the effectiveness of various input signals for power system stabilizers. Proc. Sixth National Power System Conference (New Delhi: Tata McGraw Hill)

Minto K D 1985 Design of reliable control systems: theory and computations. Ph D thesis, Department of Electrical Engineering, University of Waterloo, Canada

Munro N, Hirbod S N 1979 Pole assignment using full rank output feedback compensators. Int. J. Syst. Sci. 10: 285-306

Padiyar K R, Prabhu S S, Anwar A 1986 Effectiveness of control signals for power system stabilizers. Proc. IFAC Symposium on Automation and instrumentation for Power Plants (Bangalore: Inst. Eng.)

Sen Gupta D P 1989 Dynamic stability in electric power systems. J. Inst. Eng. (India) J-EL 70: $117-124$

Sen Gupta D P, Narahari N G, Boyd I, Hogg B W 1985 An adaptive power system stabilizer which cancels the negative damping torque of a synchronous generator. Proc. Inst. Electr. Eng. 132: 109-117

Sharma L N 1991 Coordination of PSS and SVC stabilizers based on robustness consideration. $\mathrm{M}$ Tech thesis, Department of Electrical Engineering, Indian Institute of Technology, Kanpur 